\title{
Will patient-centric sampling become the norm for clinical trials after COVID-19?
}

To the Editor - Patient-centric sampling, or the practice of self-collection by patients or the collection of samples remotely from clinical facilities, offers obvious benefits. This approach can increase the efficiency and speed of clinical trials and allow additional sampling time points. It also reduces patient burden and provides opportunities for wider and more-diverse participation in clinical trials. There are multiple examples of the successful application of patient-centric sampling for diagnostic testing and in clinical trials ${ }^{1-6}$. However, adoption of patient-centric sampling in clinical trials has been slow, in part because of ongoing questions about the quality of sampling, sample stability and comparability to standard sampling methods. However, in the midst of the COVID-19 pandemic restrictions, patient-centric sampling has often been the only option for diagnostic testing and disease tracking, which has propelled this approach into the mainstream.

Several patient-centric sampling approaches have been employed in the context of COVID-19 testing. These include drive-through testing sites, deployment of nurses to collect samples in patients' homes, and at-home self-collection. More than 20 molecular diagnostic tests for the coronavirus SARS-CoV-2 with home collection have been granted Emergency Use Authorization by the US Food and Drug Administration (https://www.fda. gov/medical-devices/coronavirus-disease2019-covid-19-emergency-use-autho rizations-medical-devices/ vitro-diagnostics-euas).

During the ongoing pandemic, regulatory authorities have quickly provided sponsors with guidance to address challenges for ongoing clinical trials ${ }^{7,8}$. Changes, such as the use of telemedicine ${ }^{9}$, foregoing non-essential visits and laboratory evaluations, and using local facilities for exams, diagnostic tests and sample collection, have enabled many trials to proceed while protecting patient safety and limiting exposure to COVID-19. Clinical studies using at-home blood sampling to track the prevalence of antibodies to COVID-19 are already ongoing, including a 15,000-participant study sponsored by the US National Institutes of Health (ClinicalTrials.gov identifier, NCT04334954 $)^{10}$. Another example is the CovidWatch study, in which the Tasso device is used for monthly blood sampling, together with weekly nasal swabs (https:// www.fredhutch.org/en/research/divisions/ vaccine-infectious-disease-division/ research/infectious-disease-sciences/ covid-watch.html).

In ongoing clinical trials, pivoting to full use of patient-centric sampling methods was prevented by the rigorous validation needed for complex pharmacokinetic and biomarker analysis. Pharmacokinetic data can also be affected if collection times are not accurately recorded during in-home sample collection ${ }^{5}$, and the quality of self-collected samples can also raise concerns. However, these sampling challenges can potentially be overcome with rigorous patient training and by the use of sample-collection devices that make sample collection easier and more convenient and that improve accuracy of data collection (e.g., automatic time stamping). Some of these novel devices, which allow the collection of samples at home or at remote locations ${ }^{1-4}$, have already been successfully used in phase 3 clinical trials ${ }^{5,6}$.

The COVID-19 pandemic has further demonstrated that patient-centric sampling can be implemented quickly and can provide improved safety for patients and healthcare workers while allowing large-scale collection of diagnostic and public-health data. This experience will hopefully accelerate the adoption of patient-centric sampling techniques in clinical trials.

Christopher A. James (D) $1 \bowtie$,

Matthew D. Barfield², Katie F. Maass (D) 3 , Shefali R. Patel ${ }^{4}$ and Melanie D. Anderson ${ }^{5}$ ${ }^{1}$ Amgen Research, Thousand Oaks, CA, USA. ${ }^{2}$ Roche, Basel, Switzerland. ${ }^{3}$ Genentech, South San Francisco, CA, USA. ${ }^{4} J a n s s e n$ ReD, Spring House, PA, USA.

${ }^{5}$ Merck, Kenilworth, NJ, USA.

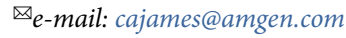

Published online: 23 November 2020 https://doi.org/10.1038/s41591-020-01144-1

References

1. Roadcap, B. et al. Bioanalysis 12, 893-904 (2020).

2. Vethe, N. T. et al. Ther. Drug Monit. 41, 607-614 (2019).

3. Kovac, J. et al. J. Pharm. Biomed. Anal. 151, 339-346 (2018).

4. Xing, J., Loureiro, J., Patel, M. T., Mikhailov, D. \& Arkady, I. Bioanalysis 12, 919-935 (2020).

5. Li, C. et al. J. Clin. Pharmacol. 58, 294-303 (2018).

6. Dockendorf, M. F. et al. https://www.simulations-plus.com/reso urce/a-model-based-approach-to-bridging-plasma-and-driedblood-spot-concentration-data-for-phase-3-verubecestat-trials/ (2018).

7. US Food and Drug Administration. https://www.fda.gov/ regulatory-information/search-fda-guidance-documents/ fda-guidance-conduct-clinical-trials-medicalproducts-during-covid-19-public-health-emergency (2020).

8. European Medicines Agency. https://ec.europa.eu/health/sites/ health/files/files/eudralex/vol-10/guidanceclinicaltrials_covid19 en.pdf (2020).

9. Hollander, J. E., \& Carr, B. G. N. Engl. J. Med. 382, 1679-1681 (2020).

10. Klumpp-Thomas, C. et al. medRxiv https://doi. org/10.1101/2020.05.21.20109280 (2020).

\section{Acknowledgements}

All authors are members of the Patient Centric Sampling Working Group of the International Consortium for Innovation and Quality in Pharmaceutical Development (IQ).

Competing interests

The authors declare no competing interests. 